\title{
Brillouin Gain Characterization by Cavity Ringdown Spectroscopy
}

\author{
Ananthu Sebastian, Stéphane Trebaol, Pascal Besnard \\ Univ Rennes, CNRS, Institut FOTON - UMR 6082, F-22305 Lannion, France
}

Nowadays, narrow linewidth lasers are unavoidable tools for fundamental and applied physics. One promising approach to generate coherent laser emission is based on stimulated Brillouin scattering (SBS) optical nonlinearity [1]. Implementing SBS in an optical cavity gives rise to coherent emission with impressive performances in terms of noise and linewidth [2]. Cascading the SBS process can even, under given conditions, improves the coherent emission specifications [3]. To achieve such performances it is crucial to determine the Brillouin gain coefficient. Usual pump-probe methods are carried out in single pass waveguides [4]. The probe intensity is proportional to $e^{g_{B} P_{i n} L_{e f f} / A_{\text {eff }}}$ where $P_{i n}$ is the incident pump power, $A_{\text {eff }}$ the effective mode area and $L_{e f f}$ the usual effective interaction length. To observe significant probe amplification one needs either long waveguides or strong pump power to reach the SBS threshold. We propose a method, based on the cavity ringdown spectroscopy, to directly characterize the Brillouin gain coefficient inside a resonator. This configuration allows to extract Brillouin parameters from the light recirculation with both a reduced waveguide length and input pump power when compared to usual methods.
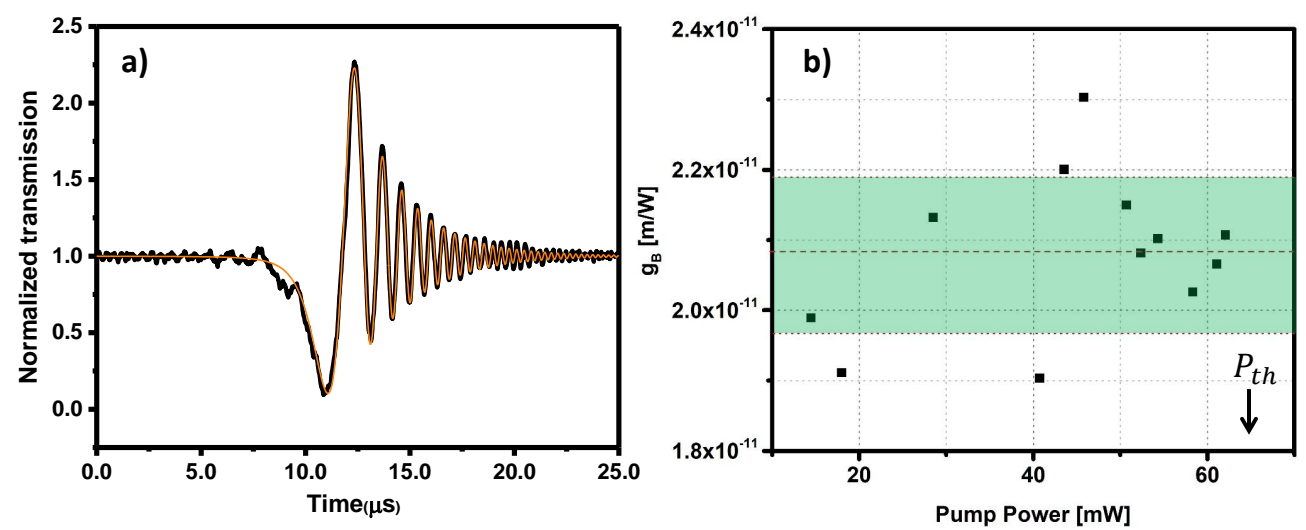

Fig. 1 a) CRDS signature obtained for a pump power delivering $43.5 \mathrm{~mW}$ (black curve) fitted by the model describes elsewhere [5] (orange curve). b) Brillouin gain coefficient extracted from the CRDS signal for various input pump powers. The mean value is equal to $g_{B}=2.08 * 10^{-11} \pm 1.1 * 10^{-12} \mathrm{~m} / \mathrm{W}$.

The CRDS has been already applied to various configurations [5]. Here, we propose to extend the domain of application to the characterization of the Brillouin gain coefficient. The principle consists in probing the transient response of the cavity mode located below the Brillouin gain curve by using a tunable narrow laser. The probe cavity is a 20-meter polarization-maintaining silica-fiber ring-resonator. During the measurement, the input pump power is adjusted to operate the resonator above the SBS threshold but below the lasing one. Fig. 1a) shows the fit of an experimental transient response with a simple model [5] that give access to the intrinsic photon lifetime $\tau_{0}$, which is related to the gain (or losses) inside the cavity, and to the extrinsic photon lifetime $\tau_{e}$, which is related to the coupling ratio of the resonator-output-coupler. $\tau_{0}$ is linked to the intensity round-trip attenuation $a^{2}=1-2 \tau_{L} / \tau_{0}$, where $\tau_{L}$ is the round trip time. An estimation of the Brillouin gain coefficient is then deduced: $g_{B}=\frac{A_{e f f}}{P_{i n} L} \ln \frac{a^{2}}{a_{o p}^{2}}$, with $a_{o p}^{2}$ the round-trip attenuation of the cold cavity.

Increasing the input pump power until the lasing threshold allows to obtain various estimation of the Brillouin gain coefficient as shown on Fig. 1b). By this technique, we obtain a mean value for the silica fiber Brillouin gain of $2.08 \times 10^{-11} \mathrm{~m} / \mathrm{W}$ with a standard deviation (shaded green region) of $\pm 1.1 * 10^{-12} \mathrm{~m} / \mathrm{W}( \pm 5,2 \%)$ being of the same order than usually reported value for Brillouin gain in silica fibers [4].

\section{References}

[1] E. P. Ippen and R.H. Stolen, "Stimulated Brillouin scattering in optical fibers", Appl. Phys. Lett. 21, pp. 539-540 (1972),

[2] R. G Smith, "Optical power handling capacity of low loss optical fibers as determined by stimulated Raman and Brillouin scattering," Appl. Opt. 11, 2489-2494 (1972)

[3] Ananthu Sebastian, Irina V Balakireva, Schadrac Fresnel, Stéphane Trebaol and Pascal Besnard, "Relative intensity noise in a multi-Stokes Brillouin laser", Optics Express, 26, pp. 33700-33711, (2018)

[4] Marc Niklès, Luc Thévenaz and Philippe Robert, "Brillouin Gain Spectrum Characterization in Single-Mode Optical Fibers", Journal of Lightwave Technology, 15, pp. 1842-1851 (1997).

[5] Yannick Dumeige, Stéphane Trebaol, Laura Ghisa, Thi Kim Ngân Nguyên, Hervé Tavernier, and Patrice Féron. "Determination of coupling regime of high-Q resonators and optical gain of highly selective amplifiers" J.Opt. Soc. Am. B 25, pp. 2073-2080 (2008) 